\title{
Robust attractor of non-twist systems
}

\author{
R. Egydio de Carvalho ${ }^{\mathrm{a}, *}$, C. Vieira Abud ${ }^{\mathrm{b}, \mathrm{c}}$ \\ a Universidade Estadual Paulista-UNESP, Rio Claro, SP, Brazil \\ ${ }^{\mathrm{b}}$ Universidade Federal de Goiás-UFG, Catalão, GO, Brazil \\ ${ }^{\mathrm{c}}$ Universidade de São Paulo-USP, São Paulo, SP, Brazil
}

\section{H I G H L I G H T S}

- We identified numerically a new kind of attractor from a shearless curve.

- The robustness of the shearless curve is carried to the dissipative case.

- The shearless curve became an attractor.

- The new attractor can be quasi-periodic or chaotic.

- The labyrinthic non-twist standard map is the model we considered.

\section{A R T I C L E I N F O}

\section{Article history:}

Received 1 August 2015

Available online 18 August 2015

\section{Keywords:}

Non-twist map

New attractor

Indicator points

Shearless

Shrimps

\begin{abstract}
A B S T R A C T
We present a new kind of one-dimensional attractor, which has not yet been predicted in the non-linear dynamics theory. We consider a non-linear map, which presents typical non-twist manifestations, as isochronous resonances and shearless torus. It is known that this torus corresponds to a very sturdy barrier in the phase space of some area-preserving systems. We show that when dissipation is present in the system, the shearless curve carries its robustness to the dissipative scenario. It becomes a powerful attractor, which we call shearless attractor, which is persistent under the variation of the parameters and it exchanges its stability from chaotic to quasi-periodic, or vice-versa, depending on the set of parameters.
\end{abstract}

(C) 2015 Elsevier B.V. All rights reserved.

\section{Introduction}

Shearless curves constitute robust transport barriers corresponding to non-twist invariant tori of nonlinear systems. Theoretical results on nonlinear dynamics associate the shearless tori [1,2] with the extremes of the winding number $[1,3,4]$. The same association occurs in confinement of plasma in tokamaks in which the shearless tori play a leading role $[5,6]$, especially due to the formation of internal transport barriers surrounding them [7,8]. It is reported that in experiments on fluid dynamics they occur when the waves have a maximum velocity [9].

A shearless torus can arise in conservative systems, in which the frequencies are locally degenerate. Such systems are usually called non-twist [10,11], or non-monotonic twist maps [12] or zero-dispersion systems [13]. Around the shearless torus some non-twist manifestations are observed as isochronous resonances [14] and separatrix reconnection $[1,2,5,15]$. When the dynamics is chaotic the separatrix reconnection is appropriately named as manifolds reconnection [16]. Close to the shearless curve there are secondary resonances with small widths which cause a kind of screening reinforcing its

\footnotetext{
* Correspondence to: Univ. Estadual Paulista-UNESP, Av. 24A, No. 1515, Bela Vista, 13506-900 Rio Claro, SP, Brazil. Tel.: +55 1935269000.

E-mail addresses: regydio@rc.unesp.br (R.E. de Carvalho), cabud@ufg.br (C.V. Abud).
} 
remarkable stability [17]. Hence, an intriguing question naturally emerges: what about the shearless curve when dissipation is introduced in the system?

For our purpose, initially we will consider, a non-dissipative, two-dimensional non-twist map, with two isochronous resonances. The shearless curve will be identified in the phase space through some symmetry properties. Next the dissipation parameter is made non-null in order to investigate the behavior of the shearless torus under the effect of such dissipation.

Some correlated works have already reported results on degenerate dissipative systems $[11,18,19]$, however the role that the shearless torus plays in the presence of dissipation remains a subject to be investigated. Our main contribution in this current work is to show the persistence of the robustness of the ex-shearless torus when dissipative effects are present. We show the onset of an attractor, with peculiar characteristics, which is descendant of the conservative shearless torus. It is a new and generic kind of attractor and we call it as shearless attractor (SA).

The paper is organized as follows. Section 2 is devoted to present the model, the indicator numbers [20] and the shearless torus in the non-dissipative case. In Section 3 we describe the shearless attractor and its characteristics. The Lyapunov exponent analysis is presented is Section 4, while further discussions and the conclusion are in Section 5.

\section{The model and the shearless torus}

The model we will consider is the dissipative labyrinthic non-twist standard map (LNSM) [21,22], which is a perturbation of the non-twist standard map [23],

$$
\mathrm{M}:\left\{\begin{array}{l}
y_{n+1}=(1-\gamma) y_{n}-b \sin \left(2 \pi x_{n}\right)-b \sin \left(\eta 2 \pi x_{n}\right) \\
x_{n+1}=x_{n}-a\left(y_{n+1}-r_{1}\right)\left(y_{n+1}-r_{2}\right) .
\end{array}\right.
$$

The second sine has a parameter, $\eta$, that induces saddle-node bifurcations inside the resonance islands which lead to a richer dynamics. The parameters $r_{1}$ and $r_{2}$ are the roots of the non-twist term and define the positions where the two isochronous resonances will born in the phase space. The parameter $a$ interferes in the amplitudes of the islands allowing the overlap of the resonances while $b$ is the perturbation parameter which allows the breakdown of the invariant structures and the increasing of the chaotic sea. The parameter $\gamma \in[0,1)$ turns on the dissipation; if $\gamma=0$ no dissipative effect is present. The map is $2 \pi$-periodic, however the variable $\mathrm{x}$ is multiplied by $2 \pi$ in such way the period becomes unitary. This map is non-twist because it violates the twist condition $\frac{\partial x_{n+1}}{\partial y_{n}} \neq 0$.

Similarly to the standard non-twist map, the LNSM presents some symmetry properties, which will be useful to find the shearless curve when $\gamma=0$. The non-dissipative LNMS can be decomposed in two involution maps, named $\mathrm{I}_{0}$ and $\mathrm{I}_{1}$. However, for simplicity we will keep the parameter $\gamma$ in our calculations given by,

$$
\begin{aligned}
& \mathrm{I}_{0}:\left\{\begin{array}{l}
x_{n+1}=-x_{n} \\
y_{n+1}=(1-\gamma) y_{n}-b \sin \left(2 \pi x_{n}\right)-b \sin \left(\eta 2 \pi x_{n}\right)
\end{array}\right. \\
& \mathrm{I}_{1}:\left\{\begin{array}{l}
x_{n+1}=-x_{n}+a\left(y_{n+1}-r_{1}\right)\left(y_{n+1}-r_{2}\right) \\
y_{n+1}=y_{n} .
\end{array}\right.
\end{aligned}
$$

An involution is a bijection, what means that two successive applications of an involution map produce the identity. So, $\mathrm{I}_{0}^{2}=\mathrm{Id}$ as well as $\mathrm{I}_{1}^{2}=\mathrm{Id}$, where Id stands for identity. This can be easily checked by substituting the current value of $x_{n+1}$ and $y_{n+1}$ in both equations of the respective involution map and the results will be $x_{n+1}=x_{n}$ and $y_{n+1}=y_{n}$. Hence, the non-dissipative LNSM can be described as the application $\mathrm{M}=\mathrm{I}_{1} \circ \mathrm{I}_{0}$ in which one sets the current values of the variables of $\mathrm{I}_{0}$ into the corresponding variables of $\mathrm{I}_{1}$. The relevance of this decomposition has been well established for the non-twist standard map in Refs. [1,24], where the authors show that the involution maps $\mathrm{I}_{0}$ and $\mathrm{I}_{1}$ have reverse orientation and their fixed points constitute one-dimensional sets which they called symmetry lines, and as reported in Refs. [25,26], the existence of symmetry lines allows to find symmetric periodic orbits. Due to the violation of the twist condition, at least two chains of periodic orbits appear with the same rotation number on each symmetry line. In addition, it is shown in Ref. [2] that:

(i) non-twist standard-like maps have invariance under the operation $S$ of the cylinder, which is indeed, a spatial symmetry defined by $x$, with unitary period, as $\mathrm{S}=(x+1 / 2,-y)$ where $\mathrm{S}$ is an involution, $\mathrm{S}^{2}=$ Id. This invariance can be easily verified through $\mathrm{M}=\mathrm{S}^{-1} \mathrm{M} \mathrm{S}$;

(ii) due to the S-symmetry, Poincaré-Birkhoff chains may be replicated and, points of the map follow the relation $\left(x_{n}, y_{n}\right)=$ $\left(x_{n}+1 / 2,-y_{n}\right)$. Hence S-symmetric orbits have the same rotation number and the corresponding island chains are in opposite side of the shearless;

(iii) the map is time-reversal symmetric with respect $\mathrm{I}_{0}, \mathrm{I}_{1}$ and $\mathrm{S}$;

(iv) the involutions $\mathrm{I}_{0}$ and $\mathrm{I}_{1}$ are orientations reversing while $\left(\mathrm{S} \circ \mathrm{I}_{0}\right)$ and $\left(\mathrm{S} \circ \mathrm{I}_{1}\right)$ are orientation preserving;

(v) the involutions $\mathrm{I}_{0}, \mathrm{I}_{1}$ and $\mathrm{S}$ generate a group and their products are members of the group;

(vi) there exists a curve which is invariant under the action of $\left(S \circ I_{0}\right)$ or $\left(S \circ I_{1}\right)$. Such curve is denoted as shearless curve in Ref. [1];

(vii) as a consequence of the invariance cited above, the fixed points of $\left(S \circ \mathrm{I}_{0}\right)$ and $\left(\mathrm{S} \circ \mathrm{I}_{1}\right)$ always belong to the shearless curve and they were called as indicator points in Ref. [1]. 

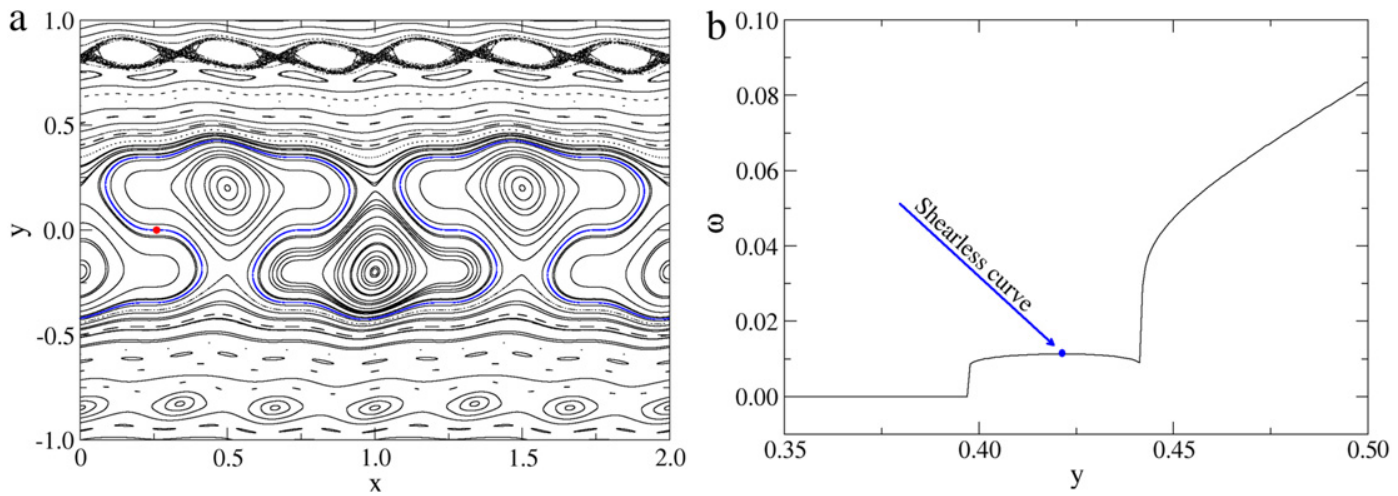

Fig. 1. (a) Typical phase space of the non-dissipative LNSM for $a=0.5, b=0.02, r_{1}=-r_{2}=0.2$ and $\eta=3$. The shearless curve is in blue and was obtained from iterations of the red point; (b) Profile of the corresponding winding number. The maximum point of the non-monotonic profile represents the shearless curve. (For interpretation of the references to color in this figure legend, the reader is referred to the web version of this article.)

As the non-dissipative LNSM is a non-twist standard-like map it is straightforward to observe that the application $\mathrm{S}$ commutes with $\mathrm{I}_{1}$ and will commute with $\mathrm{I}_{0}$ for $\eta$ odd so $\left(\mathrm{S} \circ \mathrm{I}_{0}\right)$ and $\left(\mathrm{S}^{\circ} \mathrm{I}_{1}\right)$ are also involutions and the non-dissipative LNSM can be rewritten as $\mathrm{M}=\left(\mathrm{S} \circ \mathrm{I}_{0}\right)\left(\mathrm{S} \circ \mathrm{I}_{1}\right)$. In that sense, the fixed points $\left(x^{*}, y^{*}\right)$ of $\left(\mathrm{S} \circ \mathrm{I}_{0}\right)$ and $\left(\mathrm{S} \circ \mathrm{I}_{1}\right)$ belong to a shearless torus and we denote them respectively as the indicator points $\left(\mathrm{IP}_{0}\right)_{1}=\left(\frac{1}{4}, \frac{b}{2}\left[1+(-1)^{\frac{\eta-1}{2}}\right]\right)$, $\left(\mathrm{IP}_{0}\right)_{2}=$ $\left(\frac{3}{4}, \frac{b}{2}\left[-1+(-1)^{\frac{\eta+1}{2}}\right]\right)$; and $\left(\mathrm{IP}_{1}\right)_{3}=\left(\frac{1}{4}+\frac{a r_{1} r_{2}}{2}, 0\right),\left(\mathrm{IP}_{1}\right)_{4}=\left(\frac{3}{4}+\frac{a r_{1} r_{2}}{2}, 0\right)$. We point out that the indicator points occur in maps that presents spatial symmetry and are time-reversal [2].

In order to verify the shearless we introduce the winding number, $\omega$, which is defined, for a regular orbit, as the limit, $\omega=\lim _{n \rightarrow \infty} \frac{\left(x-x_{0}\right)}{n}$, where $x$ is kept unbounded, for rotation tori $[1,27]$ and $x_{0}$ is an initial value. If the winding number is a rational number it describes a periodic orbit while if it is an irrational it represents a quasi-periodic one. The winding number does not converge for chaotic orbits. When the winding number profile has extremes, they correspond to invariant tori which are identified as the shearless tori $[22,24]$ and for each reconnection process, a region of meandering tori can appear and one of them is the shearless curve [22].

In Fig. 1(a), we observe the dynamics in the phase space considering the parameters $\gamma=0, b=0.02, r_{1}=0.2=-r_{2}$ and we plot the shearless torus, which is shown in blue, from the $\left(\mathrm{IP}_{1}\right)_{3}$ which is marked with a red point. Fig. 1(b) shows the winding number of the orbits from the line of initial conditions $x=0.5, y \in[0.35: 0.50]$. For each initial value $y_{0}$, we storage $\left(y_{0}, \omega\right)$; the shearless torus is identified with a blue dot in the extreme of the winding number.

In Fig. 2 we set the parameter $b=0.056$ and keep all the others as depicted in Fig. 1. We observe that many invariant tori are destroyed but the shearless curve, in blue, still survives, it was generated from the same point $\left(\mathrm{IP}_{1}\right)_{3}$. We see two distinct chaotic regions separated by the shearless torus, the initial conditions started in the red or black regions do not cross the shearless barrier.

It is worth noting that the chain with the void island in Fig. 2, seems to be the same type reported in Ref. [28].

\section{The "shearless" attractor}

A remarkable characteristic of the shearless torus is its robustness under the effect of perturbations because its winding numbers is not well approximate by rational numbers. Since a shearless torus survives for relatively strong perturbations and lives together with the chaotic sea, an intriguing question naturally emerges on what will globally happen in the system with the introduction of dissipation when there is at least one shearless torus. In order to understand this point, we explored numerically the evolution of the LNSM for different intensities of the parameter of dissipation. For all calculations we fixed $a=0.5, \eta=3, r_{1}=0.2=-r_{2}, b=0.056$ and changed $\gamma$ appropriately.

In Fig. 3, we show the evolution of the attractors for the dissipative LNSM. The plots have been obtained by iterating $10^{4}$ times a set of initial conditions and plotting only the last hundred iterations. In Fig. 3(a) we set $\gamma=0.001$ and we observe point attractors and a curve very similar to the shearless curve of the non-dissipative case. The plot of Fig. 3(b) also presents this shearless curve and point attractors that still have survived to the dissipation for $\gamma=0.0022$. They correspond to the elliptic fixed points of the main islands when $\gamma=0$. However, several orbits that started below or above the shearless curve accumulated in this remaining topology of the shearless torus showing clearly that it has survived to the perturbation and became an attractor. Even though there is not properly a shearless torus in the dissipative case, we will link this new attractor with its origin by naming it as shearless attractor. In Fig. 3(c) with $\gamma=0.022$ we see that the shearless attractor has suffered a bifurcation and its structure is similar to a chaotic attractor. However, increasing the dissipation to $\gamma=0.05$, Fig. 3(d), the shearless attractor seems to be again quasi-periodic and it coexists with the four point attractors.

In order to characterize the shearless attractor, we calculate the associate Lyapunov exponent. Two dimensional maps have two Lyapunov exponents however to classify the attractor it is sufficient to know the largest one. So, to define the type 


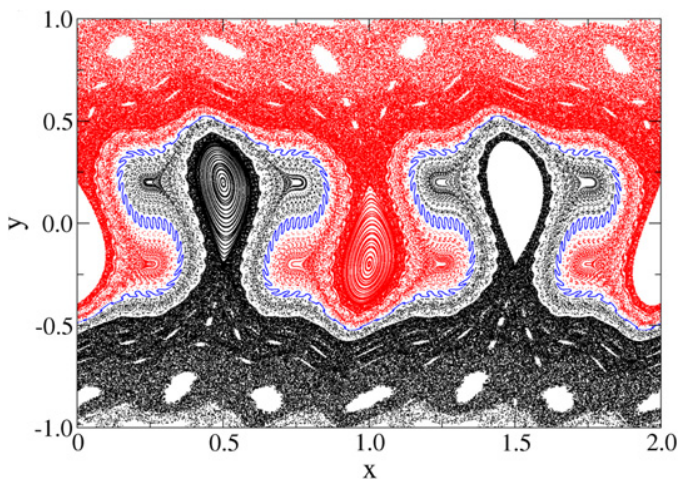

Fig. 2. Two disconnected chaotic regions separated by the shearless torus, in blue. The value of the parameter of perturbation is $b=0.056$ and $\gamma=0$ (non-dissipative). (For interpretation of the references to color in this figure legend, the reader is referred to the web version of this article.)
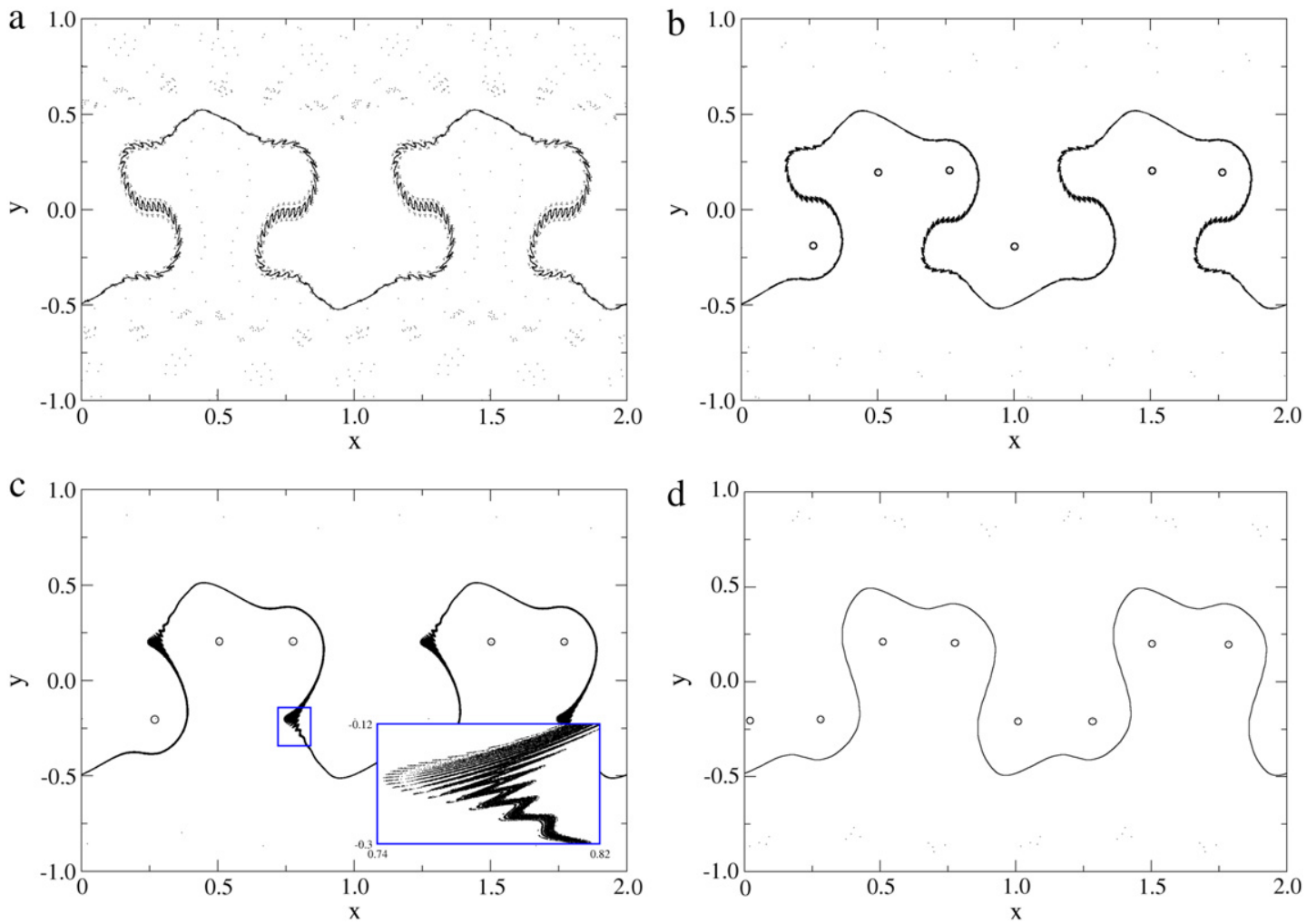

Fig. 3. Evolution of the shearless attractor, in (a), (b) and (d) the attractor is quasi-periodic while in (c) it spreads and looks like a chaotic attractor. (For interpretation of the references to color in this figure legend, the reader is referred to the web version of this article.)

of attractor that is represented in Fig. 3(c) and (d) we will calculate the maximum Lyapunov exponent. We have obtained $(\lambda)_{\max }^{c}=0.081$ and $(\lambda)_{\max }^{d}=0$, what means that the shearless attractor in Fig. 3(c) is chaotic and the one in Fig. 3(d) is quasi-periodic.

This result shows us that the shearless attractor has bifurcated by varying the dissipation parameter and so another question emerges: Is this bifurcation unique? However, before dealing with this point we present in Fig. 4 the basins of attraction of all attractors present in Fig. 3(c). The yellow region corresponds to the orbits that converged to the shearless attractor. Note that there are other four basins of attraction which are related to the four stable foci, two of them (black and green) are associated with orbits coming from the bottom of the phase space while the other two (red and blue) are associated with orbits that come from the top of the phase space. This plot qualitatively suggests that the shearless attractor divides the basins of attraction of the stable foci in two distinct groups. Furthermore, the shearless attractor presents a basin of attraction bigger than the one of each stable focus.

For a better understanding on what kind of attractor is the shearless attractor we need to vary the control parameters and calculate the corresponding Lyapunov exponents. The projection of the Lyapunov exponents onto the space of the 


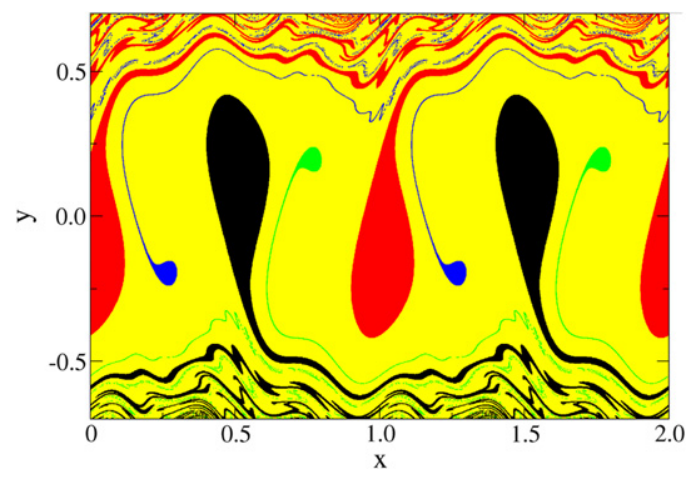

Fig. 4. Basins of attraction of each attractor present in Fig. 3(c).

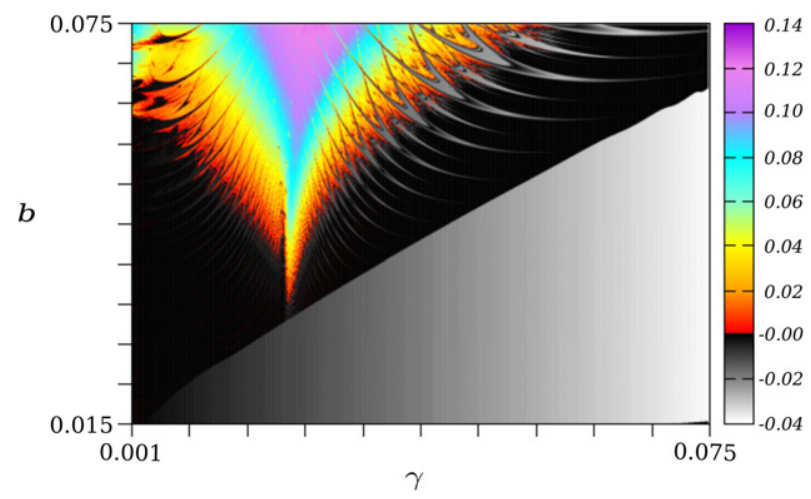

Fig. 5. Lyapunov phase diagram characterizing the shearless attractor for the LNSM with $a=0.5$. White and gray tones mean that it does not exist, the black region characterizes it as quasi-periodic and colored regions identify it as chaotic. (For interpretation of the references to color in this figure legend, the reader is referred to the web version of this article.)

parameters is called Lyapunov diagrams, which can give conclusive information about the nature of this new attractor and its bifurcations [29]. In the next section we investigate the Lyapunov exponent of the shearless attractor in the $(b \times \gamma)$ parameter space.

\section{Lyapunov diagrams}

To obtain the Lyapunov diagram for the shearless attractor from the dissipative LNMS we fixed $a=0.5$ and we divided the space parameter $(b, \gamma)$ in a grid of $1000 \times 1000$. The maximum Lyapunov exponent is calculated for each pair $(b, \gamma)$ and displayed in a palette of colors escalating. In Fig. 5 we show the Lyapunov diagram $(b, \gamma)$, whose implications are the following: white and gray tones indicate the non-existence of the shearless attractor because the orbit converged toward any stable focus $\left(\lambda_{\max }<0\right)$. The black region characterizes the shearless attractor as quasi-periodic $\left(\lambda_{\max }=0\right)$, and the colored regions, where the maximum Lyapunov exponent is positive, identify the shearless attractor as chaotic. Hence, when the shearless attractor does exist it can assume quasi-periodic or chaotic behavior and the route (quasi-periodic) $\leftrightarrow$ (chaos) of the shearless attractor depends on the complexity of the structures present in Fig. 5.

We also would like to emphasize that to obtain Fig. 5 we have used the value of the $\left(\mathrm{IP}_{1}\right)_{3}$ of the non-dissipative case as initial condition to follow the shearless attractor, what led us to conclude that when the shearless attractor does exist, the indicator points of the non-dissipative LNMS belong to its basin of attraction. It is worth point out that there are not indicator points for the dissipative LNMS. Another point that should be mentioned is the presence of the so-called periodicwindows, also known as shrimps, in the Lyapunov diagram. These remarkable structures are usual to appear in the space of parameters of dissipative non-linear dynamical systems [29-31]. In our case the shrimps correspond to regions where the shearless attractor does not exist and only the stable foci are present, or when it is quasi-periodic.

In Fig. 6 we present an amplification of Fig. 5 in order to show the fine structure of the shrimps and also the subtle transitions /bifurcations of the shearless attractor.

\section{Remarks and conclusion}

A limit cycle attractor is usually defined as an invariant set corresponding to an isolated orbit in the sense that neighboring orbits are not closed, they travel toward it. An isolated limit cycle attractor encircles an unstable fixed point. Our shearless 


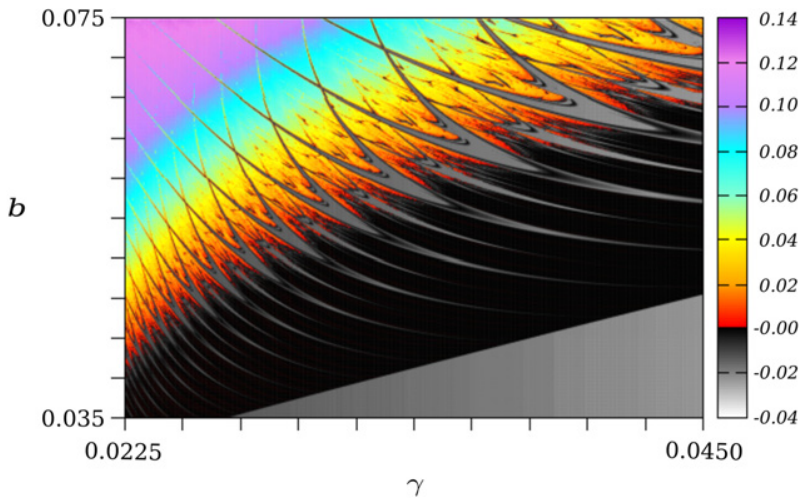

Fig. 6. Amplification of Fig. 5 in the space of parameters $(b, \gamma)$.

attractor is a x-periodic orbit but it is not closed and because that it does not encircles fixed points. Orbits in its neighborhood can travel toward it or toward the other attractors. Even though there is some similarity with the limit cycle, the shearless attractor is not a limit cycle and presents its own characteristics. Additionally, even though the shearless curve does not exist in the dissipative regime, we used its denomination in order to characterize the attractor since the shearless curve originated it.

In short, we report the occurrence of a new kind of attractor using a non-twist map which presents isochronous resonances. Among these there exists a meandering region that contains the transport barrier shearless torus. We identify the shearless from symmetry properties of the non-dissipative LNSM and we get it numerically from the corresponding indicator points. When dissipation is present in the system, this sturdy barrier becomes a powerful attractor, called shearless attractor, which can be quasi-periodic or chaotic depending on the set of parameters. We calculate the maximum Lyapunov exponents and the corresponding Lyapunov diagram in the space of parameters $(b, \gamma)$. We observed different regions in which the shearless attractor changes its nature between quasi-periodic and chaotic. We have also gotten shrimp structures in the Lyapunov diagram. We point out that as shrimps as the Lyapunov diagram refer only to the shearless attractor, the iterations have been done from an indicator point of the non-dissipative LNSM. This allows us to conclude that the value of the ex-indicator point is in the basin of attraction of the shearless attractor. These results are a new non-twist manifestation and correspond to a new fundamental for the non-linear dynamics theory which can permeate many branches of the science. Although we have used a specific map, we evaluate that there is no loss of generality in the results.

\section{Acknowledgments}

The authors thank the Brazilian scientific agency São Paulo Research Foundation (FAPESP) for financial support through the grants: 2010/20276-2, 2013/17989-5 and 2014/00334-9. CVA also thanks E. S. Medeiros for fruitful discussions.

\section{References}

[1] D. del Castillo-Negrete, J.M. Greene, P.J. Morrison, Physica D 91 (1996) 1

[2] E. Petrisor, Internat. J. Bifur. Chaos 11 (2001) 497.

[3] J.M. Greene, J. Math. Phys. 20 (1979) 1183.

[4] S. Shinohara, Y. Aizawa, Progr. Theoret. Phys. 97 (1997) 379.

[5] P.J. Morrison, Phys. Plasmas 7 (2000) 2279.

[6] C.V. Abud, I.L. Caldas, Nucl. Fusion 54 (2014) 064010.

[7] J.W. Connor, et al., Nucl. Fusion 44 (2004) R1.

[8] D. Constantinescu, J.H. Misguich, I. Pavlenko, E. Petrisor, J. Phys.: Conf. Ser. (2005) 233.

[9] R.P. Behringer, S.D. Meyers, H.L. Swinney, Phys. Fluids A 3 (1991) 43.

[10] S.H. Strogatz, Nonlinear Dynamics and Chaos: With Applications to Physics, Biology, Chemistry, and Engineering, Westview Press, 2001.

[11] P.J. Morrison, A. Wurm, Scholarpedia 4 (9) (2009) 3551

[12] J.E. Howard, J. Humpherys, Physica D 80 (1995) 256.

[13] S.M. Soskin, R. Mannella, P.V.E. McClintock, Phys. Rep. 373 (2003) 247.

[14] R. Egydio de Carvalho, A.M.O. de Almeida, Phys. Lett. A 162 (1992) 457.

[15] D. Morozov, S.A. Boykova, Regul. Chaotic Dyn. 4 (1999) 70.

[16] G. Corso, F.B. Rizzato, Phys. Rev. E 58 (1998) 8013.

[17] I.I. Ripina, M.G. Brown, F.J. Beron-Vera, H. Koak, M.J. Olascoaga, I.A. Udovdchenkov, Phys. Rev. Lett. 98 (2007) 104102

[18] A.D. Morozov, Regul. Chaotic Dyn. 9 (2004) 337.

[19] E.G. Altmann, G. Cristadoro, D. Pazó, Phys. Rev. E 73 (2006) 056201.

[20] S. Shinohara, Y. Aizawa, Progr. Theoret. Phys. 100 (1998) 219.

[21] C. Simó, Regul. Chaotic Dyn. 3 (1998) 180.

[22] C.G.L. Martins, R. Egydio de Carvalho, I.L. Caldas, M. Roberto, J. Phys. A 44 (2011) 045102.

[23] D. del Castillo-Negrete, P.J. Morrison, Phys. Fluids A 5 (1993) 948.

[24] A. Wurm, A. Apte, K. Fuchss, P.J. Morrison, Chaos 15 (2005) 023108.

[25] J.M. Greene, J. Math. Phys. 9 (1968) 760 
[26] R. de Vogelaere, in: S. Lefschetz (Ed.), Contributions to the Theory of Nonlinear Oscillations, vol. IV, Princeton University Press, Princeton, NJ, 1958, p. 53.

[27] A. Apte, R. de la Llave, N.P. Petrov, Nonlinearity 18 (2005) 1173.

[28] M.C. de Sousa, I.L. Caldas, A.M. Ozorio de Almeida, F.B. Rizzato, R. Pakter, Phys. Rev. E 88 (2013) 064901

[29] R. Vitolo, P. Glendinning, J.A.C. Gallas, Phys. Rev. E 84 (2011) 016216.

[30] E.S. Medeiros, R.O. Medrano-T, I.L. Caldas, S.L.T. de Souza, Phys. Lett. A 377 (2013) 628

[31] J.A.C. Gallas, Phys. Rev. Lett. 70 (1993) 2714. 